International Journal on Applications of Graph Theory in Wireless Ad hoc Networks and Sensor Networks(GRAPH-HOC) Vol.6, No.4, December 2014

\title{
On Algorithmic Problems Concerning GRAPHS OF HIGHER DEGREE OF SYMMETRY
}

\author{
VISHAL GOUNDAR \\ School of Engineering,New Zealand
}

\begin{abstract}
Since the ancient determination of the five platonic solids the study of symmetry and regularity has always been one of the most fascinating aspects of mathematics. One intriguing phenomenon of studies in graph theory is the fact that quite often arithmetic regularity properties of a graph imply the existence of many symmetries, i.e. large automorphism group G. In some important special situation higher degree of regularity means that $G$ is an automorphism group of finite geometry. For example, a glance through the list of distance regular graphs of diameter $d<3$ reveals the fact that most of them are connected with classical Lie geometry. Theory of distance regular graphs is an important part of algebraic combinatorics and its applications such as coding theory, communication networks, and block design. An important tool for investigation of such graphs is their spectra, which is the set of eigenvalues of adjacency matrix of a graph. Let $G$ be a finite simple group of Lie type and X be the set homogeneous elements of the associated geometry. The complexity of computing the adjacency matrices of a graph $G r$ on the vertices $X$ such that Aut $G R=G$ depends very much on the description of the geometry with which one starts. For example, we can represent the geometry as the totality of 1 cosets of parabolic subgroups 2 chains of embedded subspaces (case of linear groups), or totally isotropic subspaces (case of the remaining classical groups), 3 special subspaces of minimal module for $G$ which are defined in terms of a $G$ invariant multilinear form. The aim of this research is to develop an effective method for generation of graphs connected with classical geometry and evaluation of its spectra, which is the set of eigenvalues of adjacency matrix of a graph. The main approach is to avoid manual drawing and to calculate graph layout automatically according to its formal structure. This is a simple task in a case of a tree like graph with a strict hierarchy of entities but it becomes more complicated for graphs of geometrical nature. There are two main reasons for the investigations of spectra: (1) very often spectra carry much more useful information about the graph than a corresponding list of entities and relationships (2) graphs with special spectra, satisfying so called Ramanujan property or simply Ramanujan graphs (by name of Indian genius mathematician) are important for real life applications (see [13]). There is a motivated suspicion that among geometrical graphs one could find some new Ramanujan graphs.
\end{abstract}

\section{INTRODUCTION}

Nowadays, lots of studies on graphs are motivated by real world applications. Graphs are mathematical model for road networks, telephone networks, digital circuits in information technology. Expanders are mathematical models of networks with the expansion property: there is a positive constant $C$ such that for any set $A$ containing at most half of all points on the plane (vertices) the number of all neighbours is at least clAl. For applications (parallel computation, networking, cryptography) we need expanders with a constant $C$ as large as it is possible. It is said that the infinite family of graphs $X_{i}$ is a family of expanders with constant $c$, if every $X_{i}$ has an expansion constant $\mathrm{C}$, which does not depend on $\mathrm{i}$. Expander graphs are widely used in computer Science, in areas ranging from parallel computation to complexity theory and cryptography [16].

Applications need t-regular expanders, i.e. graphs in which each vertex is connected with exactly t neighbours. It is known that random regular graphs are good expanders. For large number of 
International Journal on Applications of Graph Theory in Wireless Ad hoc Networks and Sensor Networks(GRAPH-HOC) Vol.6, No.4, December 2014

vertices $n$, most t-regular graphs are good expanders (Sarnak, see [26]). Explicit construction of infinite families of t-regular expanders ( $t$ fixed) turns out to be difficult. Gregory Margulis [21][22] constructed the only known infinite family of expanders of bounded degree. He used the deep representation theory of semisimple groups. For real life applications it is important to construct large (not necessarily infinite) families of t-regular expanders for given $t$, because we are working with finite network.

\section{PRELIMINARIES}

\subsection{Graph Theory}

A graph is a pair $\Gamma=(V, E)$ consisting of a set $V=V(\Gamma)$, referred to as the vertex set of $\Gamma$ and a set $E=E(\Gamma)$ of 2-subsets of $V$, referred to as the edge set of $\Gamma$. That is, our graphs are undirected, without loops or multiple edges. Elements of $V$ and $E$ are called vertices (or points), and edges, respectively.

The girth of a graph $\Gamma$, denoted by $g=g(\Gamma)$, is the length of the shortest cycle in $\Gamma$. If $\Gamma$ has no cycles, the girth of $\Gamma$ is infinity.

A tree is a connected (simple) graph with no cycles. Any graph without cycles is called a forest. So, a forest is a set of disjoint trees.

The following classical statements can be found in any handbook on Graph Theory. Lemma: Every finite tree with vertices has a leaf, i.e., a vertex of valency 1 . Corollary: Every $m$-regular tree, $m \geq 2$, is an infinite graph.

Lemma: An infinite $m$-regular tree exists for each $m \geq 2$. All $m$-regular trees are isomorphic.

We can view a $\mathrm{m}$-regular tree as an incidence graph for a group incidence structure. Via such a representation, a m-regular tree becomes a familiar object for algebraists.

DEFINITION (Bipartite Graph). A bipartite graph $G=(X, Y, E)$ is a graph whose vertices are partitioned into two vertex sets, $X$ and $Y$, and every edge in $G$ joins a vertex in $\mathrm{X}$ with a vertex in $\mathrm{Y}$.

The concept of degree. The degree of a vertex is the number of edges that are incident on (or stick out of) the vertex. We will show that the sum of the degrees of all the vertices in a graph is twice the number of edges of the graph.

DEFINITION Let $G$ be a graph and $v$ a vertex of $G$. The degree of $v$, denoted $\operatorname{deg}(v)$, equals the number of edges that are incident on $\mathrm{V}$, with an edge that is a loop counted twice. The total degree of $G$ is the sum of the degrees of all vertices of $G$.

DEFINITION (Symmetric Matrix). An $n \times n$ square matrix $A=\left(a_{i j}\right)$ is called symmetric if and only if, for all $i, j=1,2, \square, n \quad a_{i j}=a_{j i}$ 
International Journal on Applications of Graph Theory in Wireless Ad hoc Networks and Sensor Networks(GRAPH-HOC) Vol.6, No.4, December 2014

DEFINITION. The adjacency matrix of $\Gamma$ is the $n \times n$ matrix $A=A(\Gamma)$ whose entries $a_{i j}$ are given by

$$
\text { aij }=\left\{\begin{array}{c}
1, \text { if } v_{i} \text { and } v_{j} \text { are adjacent; } \\
0, \text { otherwise }
\end{array}\right.
$$

If $\lambda$ is an eigenvalue of $A$, then since $A$ is real and symmetric, it follows that $\lambda$ is real, and the multiplicity of $\lambda$ as a root of the equation $\operatorname{det}(\lambda \mathrm{I}-\mathrm{A})=0$ is equal to the dimension of the space of eigenvectors corresponding to $\lambda$.

DEFINITION (Spectrum). The spectrum of a graph $\Gamma$ is the set of numbers which are eigenvalues of $A(\Gamma)$, together with their multiplicities. If the distinct eigenvalues of $A(\Gamma)$ are $\lambda_{0}>\lambda_{1}>\square>\lambda_{s-1}$, and their multiplicities are $\mathrm{m}\left(\lambda_{0}\right), \mathrm{m}\left(\lambda_{\mathrm{l}}\right), \square, \mathrm{m}\left(\lambda_{\mathrm{s}-1} 1\right)$, then we shall write

$\operatorname{Spec} \Gamma=\left(\begin{array}{llll}\lambda_{0} & \lambda_{1} & \cdots & \lambda_{s-1} \\ m\left(\lambda_{0}\right) & m\left(\lambda_{1}\right) & \cdots & m\left(\lambda_{s-1}\right)\end{array}\right)$

For example, the complete graph $\mathrm{K}_{\mathrm{n}}$ is the graph with $\mathrm{n}$ vertices in which each distinct pair are adjacent. Thus the graph $\mathrm{K}_{9}$ has adjacency matrix

and the spectrum of $\mathrm{K}_{9}$ is

$$
A=\left[\begin{array}{lllllllll}
0 & 0 & 0 & 0 & 1 & 0 & 1 & 0 & 1 \\
0 & 0 & 0 & 1 & 0 & 0 & 0 & 1 & 1 \\
0 & 0 & 1 & 1 & 1 & 0 & 0 & 0 & 0 \\
1 & 0 & 1 & 0 & 0 & 0 & 0 & 1 & 0 \\
0 & 1 & 1 & 0 & 0 & 0 & 1 & 0 & 0 \\
0 & 0 & 0 & 0 & 0 & 1 & 1 & 1 & 0 \\
0 & 1 & 0 & 1 & 0 & 1 & 0 & 0 & 0 \\
1 & 0 & 0 & 0 & 1 & 1 & 0 & 0 & 0 \\
1 & 1 & 0 & 0 & 0 & 0 & 0 & 0 & 1
\end{array}\right]
$$

Spec $\mathrm{K}_{9}=\left(\begin{array}{ccc}3 & \sqrt{3} & 0 \\ 1 & 6 & 2\end{array}\right)$

We refer to the eigenvalues of $A=A(\Gamma)$ as the eigenvalues of $\Gamma$. The characteristic polynomial $\operatorname{det}(\lambda \mathrm{I}-\mathrm{A})$ will be referred to as the characteristic polynomial of $\Gamma$, and denoted by $X(\Gamma ; \lambda)$

Suppose the characteristic polynomial of $\Gamma$ is

$X(\Gamma ; \lambda)=\lambda_{n}+c_{1} \lambda^{n-1}+c_{2} \lambda^{n-2}+c_{3} \lambda^{n-3}+\square+c_{n}$

The spectrum of a bipartite graph. A graph is bipartite if its vertex set can be partitioned into two parts $V_{1}$ and $V_{2}$ such that each edge has one vertex in $V_{1}$ and one vertex in $V_{2}$. If we order the vertices so that those in $V_{1}$ come first, then the adjacency matrix of a bipartite graph takes the form

$$
A=\left[\begin{array}{cc}
0 & B \\
B^{T} & 0
\end{array}\right]
$$


International Journal on Applications of Graph Theory in Wireless Ad hoc Networks and Sensor Networks(GRAPH-HOC) Vol.6, No.4, December 2014

If $x$ is an eigenvector corresponding to the eigenvalue $\lambda$, and $\tilde{x}$ is obtained from $\mathrm{x}$ by changing the signs of the entries corresponding to the vertices in $\mathrm{V}_{2}$, then $\tilde{x}$ is an eigenvector corresponding to the eigenvalue $-\lambda$. It follows that the spectrum of a bipartite graph is symmetric with respect to 0 .

The adjacency matrix of a bipartite graph $\Gamma$ is a matrix of kind $M_{n}=\left(\begin{array}{cc}0 & B \\ B^{T} & 0\end{array}\right)$

Eigenvalues of graph $\Gamma$ are $\pm \sqrt{\lambda}$, where $\lambda$ is eigenvalues of matrix $A_{n}=B B^{T}$.

An upper bound for the largest eigenvalue. Suppose that the eigenvalues of $\Gamma$ are $\lambda_{0} \geq \lambda_{1} \geq \square \geq \lambda_{n-1}$, where $\Gamma$ has $n$ vertices and $m$ edges.

A graph is said to be regular of degree $p$ (or $p$-regular) if each of its vertices has degree $p$.

Proposition. Let $\Gamma$ be a regular graph of degree $p$. Then:

(i) $p$ is an eigenvalue of $\Gamma$;

(ii) if $\Gamma$ is connected, then the multiplicity of $p$ is 1 ;

(iii) for any eigenvalue $\lambda$ of $\Gamma$, we have $|\lambda| \leq p$.

If $v$ and $w$ are vertices of a graph $\Gamma$, and $e=\{v, w\}$ is an edge of $\Gamma$, then we say that $e$ joins $v$ and $w$, and that $v$ and $w$ are the ends of $e$.

The number of edges of which $v$ is an end is called the degree of $v$. A subgraph of $\Gamma$ is constructed by taking a subset $S$ of $E(\Gamma)$ together with all vertices incident in $\Gamma$ with some edge belonging to $S$. An induced subgraph of $\Gamma$ is obtained by taking a subset $U$ of $V(\Gamma)$ together with all edges which are incident in $\Gamma$ only with vertices belonging to $U$. In both cases the incidence relation in the subgraph is inherited from the incidence relation in $\Gamma$. see [25]

\section{DESCRIPTION OF OBJECTS}

Let $\mathrm{G}$ be a group with proper distinct subgroups $\mathrm{G}_{1}$ and $\mathrm{G}_{2}$. The group incidence structure $\Gamma(G)=\Gamma(G)_{G_{1}, G_{2}}$ has the set of points $P=\left(G: G_{1}\right)$, and the set of lines $L=\left(G: G_{2}\right)$, and incidence relation $I: p I l, \quad p \in P, l \in L$ iff the set-theoretical intersection $p \cap l$ of cosets is nonempty.

Let us consider the bipartite graph $\Gamma(G)=\Gamma(G)_{G_{1}, G_{2}}$ of the incidence relation I. We will identify the incidence structure and related bipartite graph. If $G$ is a free product of $G_{1}$ and $G_{2}$, then $\Gamma(G)$ is an infinite tree. The graphs defined in the section below in terms of equations, can be considered as $\Gamma\left(\frac{G}{F_{i}}\right)_{G_{1}, G_{2}}$ where $\mathrm{G}=\mathrm{G}_{1}{ }^{*} \mathrm{G}_{2}$ and $\mathrm{G}_{1}, \mathrm{G}_{2}$ are an elementary abelian finite group of order $p$ and $F_{i}$ is a special filtration for $G$. 
International Journal on Applications of Graph Theory in Wireless Ad hoc Networks and Sensor Networks(GRAPH-HOC) Vol.6, No.4, December 2014

The graphs $\mathrm{D}(\mathrm{m}, \mathrm{k})$ are graphs $\Gamma(G)_{G_{1}, G_{2}}$ where $\mathrm{G}_{1}=\mathrm{G}_{2}=\mathrm{Z}_{\mathrm{m}}$ and $G=\frac{\left(Z_{m} * Z_{n}\right)}{F_{n}}$ where $\mathrm{G}$ is a factor group of the free product $Z_{m}$ with $Z_{n}$ by a special normal subgroup $F_{n}$ of finite index and $\left|F_{n} \cap G_{1}\right|=\left|F_{n} \cap G_{2}\right|=1$ (for more details see[2])

\section{CONCLUSION}

We are able to create a model in a computer, t-regular expander $\Gamma$ for given number $t$, with the size $>M$, where $M$ is a given constant and estimate the related expansion constant. Possible parameters $t$ and $M$ are restricted only by the capacity of the computer. In fact we are working with 2 families of t-regular graphs $D(t, k)[13]$ and $W_{k}(t)$ [33] where $t>2$ and $k>1$ are integers, number of vertices for each graph is $2 t^{k}$. For $t=2$ the graph is not connected. We set up the computer program which demonstrate for quite big array of pairs $(\mathrm{t}, \mathrm{k})$ that these graphs are very good expanders. In fact, we got that $c \geq \frac{1}{2}-\frac{\sqrt{t}}{2 t}$ if $\mathrm{t}$ is a prime number.

Walks via edges define natural distance on graph. Let $\Gamma^{2}$ be the graph which has same vertices with $\Gamma$ and vertices connected via edge in $\Gamma^{2}$ iff they are at distance 2 in $\Gamma$.

Our computations demonstrate that graphs $\left(\mathrm{W}_{\mathrm{k}}(\mathrm{t})\right)^{2}$ and $(\mathrm{D}(\mathrm{t}, \mathrm{k}))^{2}$ are also good expanders. The valencies or the number of neighbours of them are $t(t-1)$ where $t$ is prime and expansion constant $c$ can be bounded below as $\frac{1}{2}-\frac{3}{2(t-1)}$. We are able to find (ts) regular subgraphs on $2(t-s) t^{(k-1)}$, for $\mathrm{s}=1,2$ vertices inside of $\mathrm{D}(\mathrm{t}, \mathrm{k})$ or $\mathrm{W}_{\mathrm{k}}(\mathrm{t})$ with nice expansion properties. Such graphs can be useful in situation when $\mathrm{D}(\mathrm{t}, \mathrm{k})$ is too big to operate by computer but subgraphs have reasonable size.

Graphs $W_{k}(t)$ and $D(t, k)$ have rather different properties. For instance, if $t$ is prime then $D(t, k), k$ $>3$ does not have small cycles (of length $>k+5$ ) but $W_{k}(t)$ always has cycle of length 8 .

Anyway graphs $W_{k}(t)$ and $D(t, k)$ could be defined similarly in group theoretical terms via so called free product $F(G)$ of two copies of the finite group $G$. Our results allow us to conjecture the existence of larger class of t-regular graph (quotients of geometry of $F(G)$ which contains both families $D(t, k)$ and $W_{k}(t)$ and have same bound for expansion constant. The technique we use to estimate the expansion constant is based on mysterious connections between expansion and spectral properties of graphs.

One canonical way to retrieve the information about the graph $\Gamma$ is to generate the adjacency matrix $A$, which is the matrix whose columns and rows are labelled by vertices of the graph and entry related to vertices $u$ and $v$ contains number 1 if $v$ connected by an edge with $u$, otherwise the entry is 0 .

The array of eigenvalues of $A$ is known as spectra of graph $\Gamma$. Spectra give the important information about graphs and spectral graph theory [4][5] is one of the most important branches of graph theory. Computation of eigenvalues is itself an important problem and there is the following connection between spectral and expansion properties of graphs. It can be shown that if 
International Journal on Applications of Graph Theory in Wireless Ad hoc Networks and Sensor Networks(GRAPH-HOC) Vol.6, No.4, December 2014

$\lambda_{1}(X)$ is the second largest eigenvalue of the adjacency matrix of a t-regular graph $X$, then $c \geq \frac{t-\lambda_{1}}{2 t}$.

Thus, if $\lambda_{1}$ is small the expansion constant is large. According to the well-known result of Alon and Boppana, if $X_{n}$ is an infinite family of t-regular graphs ( $t$ fixed), then $\lim \lambda_{1}\left(X_{n}\right) \geq 2 \sqrt{t-1}$.

This statement was the motivation of the study of Ramanujan graphs among t-regular graphs. A finite t-regular graph $Y$ is called Ramanujan if, for every eigenvalue $\lambda$ of $Y$, either $|\lambda|=t$ or $|\lambda| \leq 2 \sqrt{t-1}$. So, Ramanujan graphs are in some sense, best expanders.

Explicit constructions of infinite families of Ramanujan graphs of bounded or unbounded degree is important problem of Graph Theory [17]. Just few families are known to be Ramanujan. We mention some of them. Explicit constructions of families of $t$-regular Ramanujan graphs were given by Lubotzky, Phillips and Sarnak [19] for all prime t-1. They proved that graphs defined in [22] turn out to be Ramanujan. M. Morgenstern (see [24]), generalized this result. He proved the existence of infinitely many Ramanujan graphs for every valency t of type $p^{\alpha}+1$, where $\mathrm{p}$ is an arbitrary prime and $\square$ is any positive integer. Both constructions have used the deep number theory. Our computations show that graphs $\mathrm{D}(\mathrm{t}, \mathrm{k})$ and $\mathrm{W}_{\mathrm{k}}(\mathrm{t})$ ( $\mathrm{t}$ is fixed, $\mathrm{k}$ is a parameter) are Ramanujan for $\mathrm{k}=2,3,4$.

More then that we find the close formula for elements of spectra. Thus we introduce new families of Ramanujan graph. If $\mathrm{k} \geq 5$ and $\mathrm{t}$ is a prime number, graphs are "asymptotically" Ramanujan: the second largest eigenvalue for them is $2 \sqrt{t}$ and clearly the ratio $\frac{2 \sqrt{t-1}}{2 \sqrt{t}}$ goes to 1 when $t$ is growing. We will discuss the results in more details below.

\section{Conclusion 4.1}

The first aim of research was to study spectra for several families of regular algebraically defined graphs and some special induced subgraphs of them by computational and theoretical tools. For computations a new computer package has been designed. It allowed us to generate the full data about the graph in the form of adjacency matrix and afterwards to compute the spectrum of the matrix.

The second part was to analyse the data and guess the closest formula for the second largest eigenvalue or to get a close bound for it; to investigate whether or not the families are useful for application in networking (parallel computations, cryptography and etc.).

The computer experiment have been completed for the graphs $D(m, k)$ and $W(m, k)$ for the following list of parameters: 
International Journal on Applications of Graph Theory in Wireless Ad hoc Networks and Sensor Networks(GRAPH-HOC) Vol.6, No.4, December 2014

$3 \leq m \leq 64$ for $k=2$

$3 \leq m \leq 17$ for $k=3$

$3 \leq m \leq 8$ for $k=4$

$3 \leq m \leq 5$ for $k=5$

$3 \leq m \leq 4$ for $k=6$

$m=3$ for $k=7$

They present graphically two dimension pictures presenting eigenvalues (axis $0 x$ ) and the related multiplicities (axis 0y).

Vertical lines $x= \pm 2 \sqrt{m-1}$ allow us to decide whether or not the graph is Ramanujan and if not how far the expansion properties of the graph are from the properties of a Ramanujan graph.

Example of a table below shows the eigenvalues and multiplicities of eigenvalues for the graph $\mathrm{D}(5,3)$.

\begin{tabular}{|c|l|}
\hline List of eigenvalues of matrix A & Multiplicity of eigenvalues \\
\hline 0 & 88 \\
\hline & 40 \\
\hline$\pm \sqrt{5}$ & 40 \\
\hline$\pm \sqrt{10}$ & 1 \\
\hline \pm 5 &
\end{tabular}

The analysis of the data show us interesting trigonometric properties beyond the spectra of graphs. Both families of graphs $W(m, k)$ and $D(m, k), m$ is prime, belong to a large class of graphs, which are quotients of forests related to free products of groups. If $k=2$ or 3 , equations in description of objects show us that $W(m, k)$ is same as $D(m, k)$ for all possible values of $m$. But for $k>3$ the behaviour of $W(m, k)$ and $D(m, k)$ is different. For instance, the girth of graph $D(m, k)$ is at least $k+5$ but $W(m, k)$ always has a cycle of length 8 . It is very interesting that our computations show that spectral properties of the graphs are similar.

Problems about expanders is discussed in [16]. Some of them deal with families of graphs of unbounded degree, others are about families of given degree. We will specialise the parameter $k$ step by step $(k=2, k=3, \square)$. Families $D(m, k)$ and $W(m, k)$ will depend only on degree $m$.

We notice several interesting patterns depending on arithmetical properties of the parameter $\mathrm{m}$.

Let us consider the first case when $m=p$, where $p$ is a prime number.

$k=2 \quad m=p, p$ is prime number of graph $D(p, 2)$.

Number of divisors of $p$ is 2. Number of distinct eigenvalues 5 .

Conjecture:

$\lambda^{2(p-1)}(\lambda \pm \sqrt{p})^{(p-1) p}(\lambda \pm p)$.

We may conjecture that $D(p, 2), p$ is prime form a family of Ramanujan graphs.

$k=3 \quad m=p, p$ is prime number of graph $D(p, 3)$. 
Number of divisors of $p$ is 2 . Number of distinct eigenvalues is 7 .

Conjecture:

$$
\lambda^{(p-1)(p(p-1)+2)}(\lambda \pm \sqrt{p})^{2 p(p-1)}(\lambda \pm \sqrt{2 p})^{\frac{1}{2} p(p-1)^{2}}(\lambda \pm p)
$$

We may conjecture that $D(p, 3)$, $p$ is prime form a family of Ramanujan graphs.

$k=4 \quad m=p$ where $p$ is prime number of graph $W(p, 4), p \geq 5$.

Number of divisors of $p$ is 2 . Number of distinct eigenvalues is 9 .

Conjecture:

$$
\lambda^{24\left(3 \frac{p-3}{2} p+2 \frac{p-3}{2}\right)}(\lambda \pm \sqrt{p})^{p(p-1)\left(p \frac{p-1}{2}+3\right)}(\lambda \pm \sqrt{2 p})^{\frac{3}{2} p(p-1)^{2}}(\lambda \pm \sqrt{3 p})^{\frac{p}{8}(p-1)\left(p^{2}-9\right)}(\lambda \pm p)
$$

The above conjecture is checked for $p=5,7$.

We may conjecture that $W(p, 4), p$ is prime form a family of Ramanujan graphs.

We may conjecture that we have 3 new families of Ramanujan graph.

If second largest eigenvalue is bounded away from valency, graph form a family of geometric expander in sense of Alon[1]. We may conjecture about existence of the following families of geometric expander with valency $\mathrm{m}, \mathrm{m}$ is composite.

$\mathrm{k}=2 \quad \mathrm{~m}$ is a non prime degree of graph $\mathrm{D}(\mathrm{m}, 2)$

1. $\mathrm{m}=\mathrm{pq}$ where $p, q \in\{p, q: q>p \mid p$ and $q$ is a prime number $\}$

Number of divisors of $m$ is 4 . Number of distinct eigenvalues is 9 .

Conjecture:

$$
\lambda^{2\left(p^{2}(q-1)+q^{2}(p-1)-(p-1)(q-1)\right)}(\lambda \pm \sqrt{m})^{m(p-1)(q-1)}(\lambda \pm p \sqrt{q})^{q(q-1)}(\lambda \pm q \sqrt{p})^{p(p-1)}(\lambda \pm m)
$$

2. $\mathrm{m}=\mathrm{p}^{2} \mathrm{q}$ where $p, q \in\{p, q: q>p \mid p$ and $q$ is a prime number $\}$

Number of divisors of $m$ is 6 . Number of distinct eigenvalues is 13 .

Conjecture:

$$
\begin{aligned}
& \lambda^{2\left(m^{2}-m\left(m-p^{2}\right)\left(1-\frac{1}{p}+\frac{1}{p^{2}}-\frac{1}{p^{3}}+\frac{1}{p^{4}}\right)-p(p-1)\left(p^{2}+1\right)-1\right)}(\lambda \pm p \sqrt{q})^{p(p-1) m(q-1)}(\lambda \pm p q)^{p^{3}(p-1)} \\
& (\lambda \pm p q \sqrt{p})^{p(p-1)}(\lambda \pm p \sqrt{p q})^{(p-1) p q(q-1)}\left(\lambda \pm p^{2} \sqrt{q}\right)^{q(q-1)}(\lambda \pm m)
\end{aligned}
$$

3. $\quad m=8 p$ where $p$ is a prime number different from 2. Number of divisors of $m$ is 8 . Number of distinct eigenvalues is 17 .

conjecture:

$$
\begin{aligned}
& \lambda^{\frac{21}{32} m^{2}+\frac{43}{4} m-86}(\lambda \pm 2 \sqrt{2 p})^{m\left(\frac{m}{2}-4\right)}(\lambda \pm 4 \sqrt{p})^{m(p-1)}(\lambda \pm 2 p \sqrt{2})^{32} \\
& (\lambda \pm 4 \sqrt{2 p})^{2 p(p-1)}(\lambda \pm 8 \sqrt{p})^{p(p-1)}(\lambda \pm 4 p)^{8}(\lambda \pm 4 p \sqrt{2})^{2}(\lambda \pm m)
\end{aligned}
$$

4. $\quad m=6 p$ where $p$ is a prime number different from 2 and 3 . Number of divisors of $m$ is 8 . Number of eigenvalues 17 . 
International Journal on Applications of Graph Theory in Wireless Ad hoc Networks and Sensor Networks(GRAPH-HOC) Vol.6, No.4, December 2014

conjecture:

$$
\begin{aligned}
& \lambda^{\frac{5}{6} m^{2}+7 m-42}(\lambda \pm \sqrt{m})^{m(2 p-2)}(\lambda \pm 2 \sqrt{3 p})^{m(p-1)}(\lambda \pm 3 \sqrt{2 p})^{2 p(p-1)} \\
& (\lambda \pm 6 \sqrt{p})^{p(p-1)}(\lambda \pm p \sqrt{6})^{12}(\lambda \pm 2 p \sqrt{3})^{6}(\lambda \pm 3 p \sqrt{2})^{2}(\lambda \pm m)
\end{aligned}
$$

5. $m=p^{r}, p$ is a prime number conjecture:

$$
\lambda^{2 \frac{m^{2}-1}{p+1}}(\lambda \pm m) \prod_{i=1}^{r}\left(\lambda \pm p^{r-\frac{i}{2}}\right)^{(p-1) p^{2 i-1}} \quad, p \geq 2, r \geq 2
$$

$\mathrm{k}=3 \quad \mathrm{~m}$ is a non prime degree of graph $\mathrm{D}(\mathrm{m}, 3)$

1. If $m=2 t$ where $t$ is a prime number different from 2 . Number of divisors of $m$ is 4 . Number of distinct eigenvalues is 11 .

conjecture:

$$
\begin{aligned}
& \lambda^{\frac{5}{4} m^{3}-3 m^{2}+9 m-12}(\lambda \pm \sqrt{m})^{2 m(m-2)}(\lambda \pm 2 \sqrt{t})^{m(t+1)(t-1)} \\
& (\lambda \pm 2 \sqrt{m})^{t(t-1)^{2}}(\lambda \pm t \sqrt{2})^{4}(\lambda \pm m)^{2}
\end{aligned}
$$

Now let us discuss spectral properties of graphs $N(D(m, k))$ and $N(W(m, k))$.

If $m$ is prime then these families are distance two graphs for $D(m, k)$ and $W(m, k)$ respectively. If $m$ is composite, then we may obtain $N(D(m, k))$ and $N(W(m, k))$ from distance 2 graph by throwing away edges between vertices such that difference of first coordinates of them is not invertible over $\mathbf{Z}_{m}$.

Conjectures for the characteristic polynomial of $\mathrm{N}(\mathrm{D}(\mathrm{m}, 2))$

$k=2$

1. $m=p, p$ is prime number.

Number of divisors of $p$ is 2 . Number of eigenvalues 3 .

Conjecture:

$$
\lambda^{p(p-1)}(\lambda+p)^{(p-1)}(\lambda-p(p-1))
$$

We may conjecture that we have an infinite family of Ramanujan graphs of unbounded degree.

2. $\quad m=p^{s}$ where $p$ is a prime number and $s \geq 1$.

Number of divisors of $p$ is 2 . Number of distinct eigenvalues is 3 .

Conjecture:

$$
\lambda^{m^{2}-p}\left(\lambda-\frac{(p-1) m^{2}}{p}\right)\left(\lambda+\frac{m^{2}}{p}\right)^{p-1}, \text { where } \mathrm{m} \text { is a nonprime number. }
$$

3. $\mathrm{m}=2^{\mathrm{s}} \mathrm{p}^{\mathrm{t}}$ where $p \in\{p: p \geq 3 \mid p$ is a prime number $\}$ and $s, t \geq 1$.

Number of divisors of $m$ is 4 . Number of distinct eigenvalues is 5 .

Conjecture:

$$
\lambda^{m^{2}-2 p}\left(\lambda \pm \frac{(p-1) m^{2}}{2 p}\right)\left(\lambda \pm \frac{m^{2}}{2 p}\right)^{p-1}
$$


International Journal on Applications of Graph Theory in Wireless Ad hoc Networks and Sensor Networks(GRAPH-HOC) Vol.6, No.4, December 2014

4. $\quad \mathrm{m}=\mathrm{qp}$

where $p, q \in\{p, q: p \geq 5$ and $q \geq 3|p \neq q| p>q \mid p$ and $q$ is a prime number $\}$

Number of divisors of $m$ is 4 . Number of distinct eigenvalues is 5 .

Conjecture:

$$
\lambda^{m^{2}-m}(\lambda-m(q-1)(p-1))(\lambda-m)^{(q-1)(p-1)}(\lambda+m(q-1))^{p-1}(\lambda+m(p-1))^{q-1}
$$

5. $\quad m=a^{r} b^{s} c^{t}$

where

$a, b, c \in\{a, b, c: a \geq 2, b \geq 3$ and $c \geq 5|\mathrm{a} \neq \mathrm{b} \neq \mathrm{c}| \mathrm{c}>\mathrm{b}>\mathrm{a} \mid \mathrm{a}, \mathrm{b}$ and $c$ is a prime number $\}$

$\mathrm{r}, \mathrm{s}, \mathrm{t} \geq 1$

Number of divisors of $\mathrm{m}$ is 8 . Number of distinct eigenvalues is 17 .

Conjecture:

$$
\lambda^{m^{2}-6 p}(\lambda \pm 2 m(p-1))(\lambda \pm m(p-1))^{2}(\lambda \pm 2 m)^{p-1}(\lambda \pm p)^{2(p-1)}
$$

Conjectures for the characteristic polynomial of $N(D(m, 3)$

$\mathrm{k}=3$

1. $\mathrm{m}=\mathrm{p}, \mathrm{p}$ is prime number.

Number of divisors of $p$ is 2. Number of distinct eigenvalues is 4 .

Conjecture:

$\lambda^{2 p(p-1)}(\lambda+p)^{(p-1)\left(p \frac{p-1}{2}+1\right)}(\lambda-p)^{\frac{1}{2} p(p-1)^{2}}(\lambda-p(p-1))$, where $\mathrm{p}$ is a prime number.

Case of bounded degree. This case is the most interesting one. Just two families of expanders are known previously (see [26]).

First, let us consider an interesting trigonometry beyond graphs $D(m, k)$ and $W(m, k)$ in case of prime degree. We notice here that all eigenvalues except $\pm m$ of our graphs can be written in the form $2 \sqrt{m} \cos \alpha$.

The distance two graphs for $D(m, k)$ and $W(m, k)$ coincide with $N(D(m, k))$ and $N(W(m, k))$.

The largest eigenvalue is $\mathrm{m}(\mathrm{m}-1)$ and all eigenvalues can be written in the form $2 m \cos \beta+\mathrm{m}$.

\section{Thus we have the following conjecture:}

If $m$ is fixed then families $D(m, k), W(m, k), N(D(m, k)), N(W(m, k))$ are families with fixed degree of expanders. Their second largest eigenvalue is bounded by $2 \sqrt{m}$ or $3 m$.

In case of small valency $(p=3)$ there is a hope to get infinite family of Ramanujan graph, or other families of expanders.

If $\mathrm{n}$ is composite, we notice the following patterns:

\section{Conjectures for the characteristic polynomial of $W(m, k)$ and $N(W(m, k))$, for $m=3$.}

$\mathrm{W}(\mathrm{m}, \mathrm{k})$

- Number of distinct eigenvalues is 7 .

For $k=3,4,5,6,7$ the characteristic polynomial of $W(m, k)$ can be written as: $\lambda^{16 \times 3^{k-3}}(\lambda \pm \sqrt{3})^{4 \times 3^{k-2}}(\lambda \pm \sqrt{6})^{2 \times 3^{k-2}}(\lambda \pm 3)^{3^{k-3}}$ 
International Journal on Applications of Graph Theory in Wireless Ad hoc Networks and Sensor Networks(GRAPH-HOC) Vol.6, No.4, December 2014

$\mathrm{N}(\mathrm{W}(\mathrm{m}, \mathrm{k}))$

Number of distinct eigenvalues is 4 .

For $\mathrm{k}=3,4,5,6,7$ the characteristic polynomial of $\mathrm{N}(\mathrm{W}(\mathrm{m}, \mathrm{k}))$ can be written as:

$\lambda^{\frac{4}{3^{k}}}(\lambda+3)^{8 \times 3^{k-3}}(\lambda-3)^{2 \times 3^{k-2}}(\lambda-6)^{3^{k-3}}$

The assumption that these patterns are valid for all $\mathrm{k}$ is wrong, because according to the result of Alon and Boppana (see introduction) for the second largest eigenvalue $\lambda_{1}(k)$ of graph $W(m, k)$ we have $\overline{\lim } \lambda_{1}(k) \geq 2 \sqrt{3-1}$.

Anyway it still can be two new infinite family of Ramanujan graphs of valency 3 (cubic graphs) and 6 respectively.

Conjecture for the characteristic polynomial of $W(m, k)$ for $m=4$.

Number of distinct eigenvalues is 9:

Conjecture:

$\lambda^{25 \times 2^{2 k-5}}(\lambda \pm 2)^{5 \times 2^{2(k-2)}}(\lambda \pm 2 \sqrt{2})^{7 \times 2^{2 k-5}}(\lambda \pm 2 \sqrt{3})^{2^{2(k-2)}}(\lambda \pm 4)^{2^{2(k-3)}}$

The above conjecture is checked for $k=4,5,6$.

We may assume that we got here an infinite family of Ramanujan graph of degree 4 (quadruple graphs). The formula above does not contradict to the Alon and Boppana theorem (see introduction).

\section{Free Product. Generalized conjecture:}

Connected components of graphs $D(m, k)$ when $k$ is even and of $W(m, k)$, can be described in group theoretical terms as graphs of incidence structure $\Gamma(G / F)_{G_{1}, G_{2}}$ (see page 33), where $\mathrm{G}_{1}$ and $\mathrm{G}_{2}$ are two copies of cyclic group $\square_{\mathrm{m}}, \mathrm{G}$ is the free product $G_{1} * G_{2}$ and $\mathrm{F}$ is a special normal subgroup. If $m$ is prime, $D(m, k)$ and $W(m, k)$ do not contain cycles $C_{4}$ and $C_{6}$ (see [12]).

\section{We may generalize this in the following conjecture:}

Let $\mathrm{G}_{1}$ and $\mathrm{G}_{2}$ be two copies of finite group $\mathrm{H}$, and let $\mathrm{F}$ be a normal subgroup of $\mathrm{G} 1{ }^{*} \mathrm{G} 2$ such that $\left|G_{1} \cap F\right|=\left|G_{2} \cap F\right|=1$ and the graph $\Gamma=\Gamma\left(G_{1} * G_{2} / F\right)_{G_{1} * G_{2}}$ has no cycles $C_{4}, C_{6}$. Then the second largest eigenvalue of $\Gamma$ is bounded by $2 \sqrt{|H|}$.

This conjecture has been presented at conferences [9][30] and specialists expressed an interest to work on the proof of it.

The problem to generate network with good expansion property (in other words expanding graphs) is very important for applications. We are able to construct some finite sequences of Ramanujan graphs of unbounded prime degree. In fact, even infinite family of graphs if our conjectures are correct. 
International Journal on Applications of Graph Theory in Wireless Ad hoc Networks and Sensor Networks(GRAPH-HOC) Vol.6, No.4, December 2014

We have construction of geometric expanders of valency $\mathrm{m}$ and $m \times \phi(m)$, $\mathrm{m}$ with special arithmetic properties. If our generalized conjecture is true, we can generate a geometrical expander of any degree $(|\mathrm{H}|$ can be any number).

Additionally, we have several families of graphs "under suspicion" that they are expanders of bounded degree. Our graphs can be computed efficiently and a specialist may use them in different problems: parallel computation, cryptography or networking.

\section{REFERENCES}

[1] N.Alon, Eigenvalues, geometric expanders, sorting in rounds and Ramsey theory, Combinatorica, 6, 83-96, 1986.

[2] H. Anton, Elementary Linear Algebra,John Wiley \& Sons, Inc., 1994.

[3] N. Biggs, Algebraic Graph Theory, Cambridge Mathematical Library

[4] D. Cvetcovic, M. Doob and H. Sachs, Spectra of Graphs, Academic Press, N.Y., 1979.

[5] D. Cvetcovic, M. Doob and H. Sachs, and A. Torgasev, Recent results in the Theory of Graph Spectra, North Holland, 1988.

[6] N. Deo, Graph Theory with Applications to Engineering and Computer Science, Washington State University, 68-69, 145-146, 1986.

[7] S. S. Epp, Discrete Mathematics with Applications (second edition), Depaul University, 369-370, 1995.

[8] J. B. Fraleigh, A First Course in Abstract Algebra (sixth edition), University of Rhode Island, 1999.

[9] V. Gounder, Free product of abelian groups and special graphs, AGRAM 2000, abstracts on international conference, University of Western Australia, Perth, Australia, 1 page, July 9-15, 2000.

[10] D. Jungnickel, Algorithms and Computation in Mathematics. In: Graphs, Networks and Algorithms, Volume 5, 13-15, 1998.

[11] F. Lazebnik, V. Ustimenko, A. Woldar, A new series of dense graphs of large girth, BulL Amer. Math. Soc., 73-79,1995.

[12] F. Lazebnik F. and V. Ustimenko, Explicit construction of graphs with an arbitrary large girth and of large size, Discrete AppL Math., 60, 275-284, 1995.

[13] F. Lazebnik and V. Ustimenko, Some Algebraic Constructions of Dense Graphs of Large Girth and of Large Size, DIMACS series in Discrete Math. and Theoret. Comput. ScL, 10, 75-93, 1993.

[14] F. Lazebnik, V.A. Ustimenko and A. J. Woldar (1995), A New Series of Dense Graphs of High Girth, Bull (new Series) of AMS, $N^{\circ} 1,32,73-79,1995$.

[15] F. Lazebnik, V.A. Ustimenko and A.J. Woldar, A characterization of the components of the graphs D(k,q), Discrete Math., 157, 271-283, 1996.

[16] A. Lubotzky, Discrete Groups, Expanding Graphs and Invariant Measures, Progr. in Math., Birkhauser, 125, 1994.

[17] A. Lubotzky, Cayley Graphs:Eigenvalues, Expanders and Random Walks, London Math. Soc., Lecture Notes, Series 218, Surveys in Combin., 155-190, 1995.

[18] A. Lubotzky and B. Weiss, Groups and Expanders, DIMACS series in Discrete Math. and Theoret. Computer Sci, 10, 95-109, 1993.

[19] A. Lubotzky, R. Philips, P. Sarnak, Ramanujan graphs, J. Comb. Theory., N², 62 -89, 115, 1989.

[20] W. Magnus, A. Karrass, D. Solitar, Combinatorial group theory, Intersci. publ., 1996.

[21] G. A. Margulis, Explicit construction of graphs without short cycles and low density codes, Combinatorica, 2, 71-78, 1982.

[22] G. Margulis, Explicit constructions of concentrators, Probl. Inform. Transmis., 10, 325-332, 1975.

[23] G. Margulis, Explicit group theoretical constructuion of combinatorial schemes and their applications to the designs of expanders and superconcentrators, Probl. Inform. Transmis., 24, 39-46, 1988.

[24] M. Morgenstern, Existence and explicit constructions of q+ I-regular Ramanujan graphs for every prime power q, J. Comb. Theory, Ser. B, 62, 44-62, 1994.

[25] J. J. Rotman, The Theory of Groups, Allyn and Bacon, Inc., 1976.

[26] P. Sarnak, Some application of modular forms, Cambridge U. Press, Cambridge, 1990. 
International Journal on Applications of Graph Theory in Wireless Ad hoc Networks and Sensor Networks(GRAPH-HOC) Vol.6, No.4, December 2014

[27] S. Sperner, Modern Algebra and Matrix Theory, Chelsea Pub.com, 1951.

[28] W. H. Teukolsky, W. T. Vetterling, B. P. Flannery, Numerical Recipies in C. Second Edition. Cambridge University Press 1988, 1992.

[29] V. A. Ustimenko, Coordinatization of regular tree and its quotients, Voronoj's Impact on Modern Science, book 2, (Proceedings of the Memorial Voronoj Conference, Mathematics and its Applications Vol 21, Institute of Mathematics, Kiev, 125-152, 1998.

[30] V. Ustimenko, V. Gounder, Proceedings of international conference on algebra and algebraic structures, Sumy, Ukraine, 111-112, June, 2001.

[31] A. Valette, Graphes de Ramanujan et applications, Seminaire Bourbaki, Nº829, 1 -29, 1997.

[32] R.F.C.Walters, Number Theory, University of Sydney, Carslaw Publications, 24-26, 1992.

[33] R. Wenger, Extremal graphs with no C4, C6 and ClOs, J. Comb. Theory, Ser. B, 52,113-116, 1991.

[34] Biggs, N.L., Lloid, E.K. and Wilson, R.J., Graph Theory 1736-1936, Clarendon Press, Oxford, 1976. 
International Journal on Applications of Graph Theory in Wireless Ad hoc Networks and Sensor Networks(GRAPH-HOC) Vol.6, No.4, December 2014

\section{INTENTIONAL BLANK}

